\title{
The Advantage of Mucus for Adhesive Locomotion in Gastropods
}

\author{
Mayuko Iwamoto ${ }^{\mathrm{a}, \mathrm{b}, *}$, Daishin Ueyama ${ }^{\mathrm{a}, \mathrm{b}, \mathrm{c}}$, Ryo Kobayashi ${ }^{\mathrm{b}, \mathrm{d}, \mathrm{e}}$ \\ ${ }^{a}$ Department of Mathematical Modeling, Analysis and Simulation, Graduate School of \\ Advanced Mathematical Sciences, Meiji University, \\ 4-21-1 Nakano, Nakano-ku, Tokyo 164-8525, Japan \\ ${ }^{b}$ Meiji Institute for Advanced Study of Mathematical Sciences, 4-21-1 Nakano, \\ Nakano-ku, Tokyo 164-8525, Japan \\ ${ }^{c}$ Department of Mathematical Sciences Based on Modeling and Analysis, Faculty of \\ Interdisciplinary Mathematical Sciences, Meiji University, \\ 4-21-1 Nakano, Nakano-ku, Tokyo 164-8525, Japan \\ ${ }^{d}$ Department of Mathematical and Life Sciences, Graduate School of Science, Hiroshima \\ University, \\ 1-3-1 Kagamiyama, Higashihiroshima, Hiroshima 739-8526, Japan \\ ${ }^{e}$ JST CREST, 5 Sanbancho, Chiyoda-ku, Tokyo 102-0075, Japan
}

\section{Abstract}

For many gastropods, locomotion is driven by a succession of periodic mus-

2 cular waves (contractions and relaxations) moving along the foot. The force

3 generated by these waves is coupled to the substratum by a thin layer of

4 pedal mucus. Gastropod pedal mucus has unusual physical properties: the

5 mucus is a viscoelastic solid at small deformation and shows a sharp yield

6 point; then, at greater strains, the mucus is a viscous liquid, although it

7 will recover its solidity if allowed to heal for a certain period. In this paper,

8 to clarify the role of the mucus and the flexible muscular waves in adhesive migration can be realized through the interaction between the periodic muscular waves and the specific physical features of mucus. Our results indicate that the hysteresis property of mucus is essential in controlling kinetic fric-

Prêpreint

November 7, 2013

(C) 2014. This manuscript version is made available under the Elsevier user license http://www.elsevier.com/open-access/userlicense/1.0/ 
tion for the realization of crawling locomotion. Furthermore, our numerical calculations show that both the hysteresis property of mucus and the contraction ratio of muscle gives rise to two styles of locomotion, direct waves and retrograde waves, which until now have been explained by different mechanisms. The biomechanical effectiveness of mucus in adhesive locomotion is also discussed.

Keywords: muscular wave, hysteresis loop, viscoelasticity, yield point, mathematical model

\section{Introduction}

Adhesive locomotion is one of the most fundamental locomotory strategies for many animals without extremities. Gastropods, including snails, slugs, and abalones, are able to crawl steadily by propagating periodic muscular waves (alternating contraction and expansion) along the ventral foot in contact with the substrate ${ }^{1-4}$. Gastropods interact with the substrate through the viscoelastic mucus released from their foot. It is generally known that gastropod pedal mucus is produced to adjust for humidity and salinity ${ }^{5}$. However, most researchers believe that the nonlinear rheological properties of mucus play an important role in locomotion ${ }^{6-9}$. Understanding the locomotory mechanism of gastropods has been an interesting endeavor for biologists and biophysicists ${ }^{10-20}$. In recent years, from the perspective of designing biomimicry robots, adhesive locomotion has become a subject of renewed interest among engineers ${ }^{21-23}$. Although physical observations and physiological knowledge of gastropod crawling locomotion both exist, the mechanisms of interaction between the muscular waves and the physical features of mucus 
remain insufficiently elucidated.

Although it has long been known that many gastropods crawl on hard substrates by actuating muscular waves along the sole of the foot, many of the earlier studies are simply records of visual observations ${ }^{24-26}$ and classifications of the waves according to their direction of propagation ${ }^{27}$. Direct waves are defined as traveling in the same direction as the movement of the animal. By contrast, retrograde waves are in the opposite direction to that in which the animal moves. Several early studies ${ }^{14,26}$ confirmed that the waves along the foot of a gastropod are concavities. Lissmann was the first to study the kinematics and dynamics of gastropod locomotion in detail, and this enabled improvements in experimental techniques ${ }^{2,13}$. He proposed that a number of forces are involved in gastropod crawling locomotion, including external sliding friction in the form of both drag and slip. The basis for understanding the frictional mechanism that enables the creation of the propulsive force was revealed in subsequent studies.

Previous research on worm-like peristaltic locomotion ${ }^{19,28-34}$ has been crucial in advancing the study of the mechanism of adhesive locomotion in gastropods. In particular, studies using a simplified mathematical model of a chain-like body in a resistive medium, including the case of a few degrees of freedom, have indicated that directional migration of the mass center of a body can be realized through nonlinear asymmetric frictional forces ${ }^{29-34}$. The frictional mechanism of earthworms that actually move by retrograde waves has been solved: anisotropic chaetae that protrude from shortened segments act in the role of spikes. The important fact in the case of earthworms is that a system with friction against the surrounding environment 
is controlled without central signals. Because this constitutes an intelligent mechanism for primitive creatures, it would be reasonable to suppose that gastropods also have an automatic system of friction control. However, what is the structure of this friction control in gastropods?

Denny was the first to reveal the rheological characteristics of the pedal mucus ${ }^{6}$. Denny and Gosline measured the rheological properties of pedal mucus, including its finite yield stress, and reached the important conclusion that the unusual viscoelastic nature of pedal mucus acts to control friction against the substratum in the generation of the animal's driving force ${ }^{6-9}$. Although the details of how the propulsive force is generated by mucus are unclear, the indication of a mechanism without a central control is invaluable.

With the advent of the 21st century, engineers began to conduct mechanical studies that support and expand on Denny's hypothesis ${ }^{21,22}$, and investigated the key features for a complex fluid to be useful in adhesive locomotion with direct waves ${ }^{23}$. Although their models sufficiently described the rheological features of mucus by putting a strong emphasis on concepts in fluid dynamics, their disadvantage is that the wave motion is prescribed independently of the underlying muscle mechanics.

The aim of this study is to prove that crawling locomotion is achieved through an interaction between the propagation of soft-body muscular waves reported by many scientists ${ }^{1,2,16,20}$ and the properties of pedal mucus reported by Denny and Gosline ${ }^{6,7}$. Hence, a simple mathematical model for crawling locomotion is proposed. This model, which describes the interaction between animals and their environment, is a close representation of the situation that occurs in nature. Our results indicate that crawling locomotion is achieved by 
this mutual interaction and that the nonlinear hysteresis property of mucus is essential to locomotion. We also find that both direct wave locomotion and retrograde wave locomotion are produced by the same mechanism. As previously shown by Tanaka et al. ${ }^{34}$, the choice of locomotory style is related to a difference in the timing of anchoring. In our model, the difference can be determined by the properties of mucus and muscle. This means that there is no need for animals to control the friction between their bodies and the substrate.

The rest of this paper is structured as follows: The next section introduces a mathematical model for crawling locomotion, and Section 3 presents the results of numerical simulations using this model. Section 4 then discusses the simulation results, draws conclusions, and introduces future work.

\section{Modeling of adhesive locomotion}

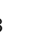

\section{t} physical properties of pedal mucus. This mechanism was implicitly suggested by Denny and Gosline ${ }^{6,7}$. Accordingly, we construct a simple mathematical model that captures the essence of this mechanism and is based on experimental results reported by Denny and Gosline ${ }^{6,7}$ amongst others ${ }^{1,2,16,26}$. Our mathematical model is considered an improvement over the model of peristaltic motion proposed by Tanaka et al. ${ }^{34}$. We now explain the details of our model. There are two critical factors in this hypothesis of the locomotory mechanism: the propagation of flexible muscular waves and the nonlinear properties of pedal mucus. 


\subsection{Muscular wave propagation}

As many scientists have reported ${ }^{1,2,10-12,16-18,26}$, muscular waves are observed during the crawling locomotion of a wide variety of mollusks. One of the main functions for self-generating a driving force is to excite a muscular contraction and then propagate the region as a wave. During locomotion, the ventral foot essentially consists of moving (slipping) regions and adhesive (anchoring) regions, with a few exceptions such as the rim of a slug. A one-dimensional model is sufficient for our aim of verifying that directional crawling locomotion is achieved.

It is reasonable to use an active spring to represent the flexible muscle of a mollusk-like gastropod. Although we might consider two types of active spring (see Appendix A), we use a real-time tunable spring (RTS) ${ }^{35}$, such as that in Figure B.1(b), to represent muscle in the mathematical model, with a view to its utilization in a robot. To mimic animals, we consider a multi-segment model, as in Figure B.1(a), which can express the propagation of muscular waves. Now, we assume that the ventral foot of the animal is divided into $(N+1)$ segments. Similar to the method of previous studies $^{23,30,33,34}$, we configure the spring to contract and elongate repeatedly. In our mathematical model, such an active spring is achieved by periodically controlling the natural length of the RTS. The natural length of the $(n+1 / 2)$ th RTS, which is one of the elements connected to the $n$th and $(n+1)$ th segments at time $t[\mathrm{~s}]$, is

$$
l_{n+\frac{1}{2}}=l_{0}(1+\alpha \sin (\omega t-\beta n)),
$$


where $\omega[\mathrm{Hz}]$ is the wave frequency. Using specific properties of the foot, we set $l_{0}[\mathrm{~m}]=L / N$ and $\beta=2 \pi W / N$, where $L[\mathrm{~m}]$ is the overall length of the foot and $W$ is the number of waves along the foot. The muscular force $F_{n+\frac{1}{2}}[\mathrm{~N}]$ acting on the $n$th segment generated by the $(n+1 / 2)$ th RTS and damper is described using $l_{n+\frac{1}{2}}[\mathrm{~m}]$ as follows:

$$
F_{n+\frac{1}{2}}=\kappa_{n+\frac{1}{2}}\left(\frac{x_{n+1}-x_{n}}{l_{n+\frac{1}{2}}}-1\right)+q_{n+\frac{1}{2}}\left(\dot{x}_{n+1}-\dot{x}_{n}\right),
$$

where $x_{n}[\mathrm{~m}]$ is the position of the $n$th segment in the direction of movement, and a dot over a variable means differentiation with respect to $t$. The first term is an elastic force generated by the RTS and the second term is the damping force. The coefficients $\kappa_{n+\frac{1}{2}}[\mathrm{~N}]$ and $q_{n+\frac{1}{2}}[\mathrm{~N} \cdot \mathrm{s} / \mathrm{m}]$ are constants. Specifically, $\kappa_{n+\frac{1}{2}}$ is the stiffness of a spring, as determined from material properties, and has the dimensions of force.

\subsection{Dynamic viscoelastic properties of pedal mucus}

The second critical factor consists of the physical properties of pedal mucus reported by Denny and Gosline ${ }^{6,7}$. Put simply, gastropod pedal mucus has two states: elastic solid and viscous liquid. The outline of Denny's experiment ${ }^{6}$ is as follows; the mucus of Pacific banana slugs (Ariolimax columbianus) was collected, and its torque against deformation was measured using a rotary cone-plate instrument for measuring viscoelasticity. First, when the strain was small, the torque was linear with respect to the deformation, as in Figure B.2(b)A. That is, the mucus behaved as an elastic solid. The mucus was found to yield when the deformation exceeded some threshold value, as in Figure B.2(b)B, and the torque became a constant independent 
of the amount of strain, as in Figure B.2(b)C. According to data reported by Denny ${ }^{6}$, this torque is linear with respect to the strain rate; that is, the mucus behaves as a viscous liquid. Thereafter, the increase in strain stops. More importantly, after a certain period of healing, the mucus recovers its elasticity, as where A follows D in Figure B.2(b).

Based on measurement results, the mucus dynamics under stress can be pictured as Figure B.3. The chemical bond that is coupled in the elastic state of the mucus breaks when the muscular contraction waves arrive. After the wave has passed through this region, the elasticity of the mucus is recovered by re-coupling of the chemical bond, although this takes a small amount of time. To describe such a thixotropic nature of mucus under the pedal, we assume that the mucus is divided into $(N+1)$, parts similar to the foot in Figure B.2(a), and define the state of the mucus by a parameter, $\sigma$; i.e., the mucus behaves as an elastic solid when $\sigma=1$ and behaves as a viscous liquid when $\sigma=0$. It then becomes clear that the pedal mucus system executes a loop, such as the one in Figure B.2(c). When the force acting on the mucus is small, the mucus is an elastic solid $(\sigma=1)$. When the force increases (A) and exceeds an upper threshold $F_{u}$, the mucus yields (B). The behavior of the mucus changes significantly, and it immediately becomes a viscous liquid $(\sigma=0)$. When the force decreases (C) below a lower threshold $F_{l}$, the mucus recovers its elasticity (D).

\subsection{Equation of motion for adhesive locomotion}

Considering the features mentioned above (muscular waves and rheology of mucus), the equation of motion for each segment can be written as follows: 


$$
m \ddot{x}_{n}=F_{n+\frac{1}{2}}-F_{n-\frac{1}{2}}-\left(1-\sigma_{n}\right) \mu \dot{x}_{n}-\sigma_{n} \gamma\left(x_{n}-\bar{x}_{n}\right),
$$

where $m[\mathrm{~kg}]$ is the mass of each segment, and the constants $\mu[\mathrm{N} \cdot \mathrm{s} / \mathrm{m}]$ and $\gamma[\mathrm{N} / \mathrm{m}]$ are the viscosity coefficient and elastic coefficient of the mucus, respectively. It is assumed that $F_{n-\frac{1}{2}}=0$ if $n=0$ and that $F_{n+\frac{1}{2}}=0$ if $n=N$. In this model, the viscoelastic behavior of mucus as friction is described by a modified Kelvin-Voigt model, where the variable $\sigma_{n}$ represents the state of the mucus under the $n$th segment. In this model, it is assumed that the critical position of the $n$th segment at the moment when the mucus changes state from a viscous liquid $\left(\sigma_{n}=0\right)$ to an elastic solid $\left(\sigma_{n}=1\right)$, as in Figure B.2(c)D, is memorized as an anchoring position $\bar{x}_{n}$. In addition, to realize the hysteresis loop shown in Figure B.2(c), the variable $\sigma_{n}$ is assumed to satisfy the following conditions:

$$
\begin{aligned}
& \text { if } \sigma_{n}=1 \text { and }\left|F_{n+\frac{1}{2}}-F_{n-\frac{1}{2}}\right|>F_{u}, \\
& \text { then } \sigma_{n} \text { changes to } 0 ; \\
& \text { if } \sigma_{n}=0 \text { and }\left|F_{n+\frac{1}{2}}-F_{n-\frac{1}{2}}\right|<F_{l}, \\
& \\
& \quad \text { then } \sigma_{n} \text { changes to } 1 \text { and } \bar{x}_{n} \text { is set to } x_{n} .
\end{aligned}
$$

Let $x_{n}=L W^{-1} \tilde{x}_{n}$ and $t=\omega^{-1} \tilde{t}$, where $\tilde{x}_{n}$ and $\tilde{t}$ are the dimensionless position and time, respectively. Then, we can obtain a system of nondimensional equations when $n \neq 0, N$ as 


$$
\begin{array}{r}
\tilde{m} \ddot{\tilde{x}}_{n}=\tilde{\kappa}\left(\frac{\tilde{x}_{n+1}-\tilde{x}_{n}}{\tilde{l}_{n+\frac{1}{2}}}-\frac{\tilde{x}_{n}-\tilde{x}_{n-1}}{\tilde{l}_{n-\frac{1}{2}}}\right)+\tilde{q}\left(\dot{\tilde{x}}_{n+1}-2 \dot{\tilde{x}}_{n}+\dot{\tilde{x}}_{n-1}\right) \\
-\left(1-\sigma_{n}\right) \tilde{\mu}_{\tilde{x}}-\sigma_{n}\left(\tilde{x}_{n}-\tilde{\tilde{x}}_{n}\right),
\end{array}
$$

where some coefficients are assumed constant, $\kappa_{n+\frac{1}{2}}=\kappa$ and $q_{n+\frac{1}{2}}=q$, and dimensionless parameters are obtained as

$$
\begin{gathered}
\tilde{m}=m \omega^{2} \gamma^{-1}, \quad \tilde{l}_{n+\frac{1}{2}}=1+\alpha \sin \left(\tilde{t}-2 \pi W \frac{n}{N}\right), \\
\tilde{\kappa}=\kappa N(\gamma L)^{-1}, \quad \tilde{q}=q \omega \gamma^{-1}, \quad \tilde{\mu}=\mu \omega \gamma^{-1} .
\end{gathered}
$$

Similarly, the two mucus thresholds (yield point and healing point) can be represented in dimensionless form as

$$
\tilde{F}_{l}=F_{l} W(\gamma L)^{-1}, \quad \tilde{F}_{u}=F_{u} W(\gamma L)^{-1}
$$

95 Now, the order of $\tilde{m}$ can be estimated at $10^{-9}$ that is vanishingly small compared with the other dimensionless coefficients, e.g., the order of the dimensionless viscosity $\tilde{\mu}$ is at $10^{-3}$, so we can assume that the inertial force is negligible (see Supplement). This assumption is reasonable in the mechanism of locomotion for gastropods and their relatives. The dimensionless equations are then:

$$
\begin{aligned}
& \left(1-\sigma_{n}\right) \mu \dot{x}_{n}+\sigma_{n}\left(x_{n}-\bar{x}_{n}\right) \\
& \quad=\kappa\left\{\frac{x_{n+1}-x_{n}}{l_{n+\frac{1}{2}}}-\frac{x_{n}-x_{n-1}}{l_{n-\frac{1}{2}}}\right\}+q\left(\dot{x}_{n+1}-2 \dot{x}_{n}+\dot{x}_{n-1}\right)
\end{aligned}
$$


where the tildes are omitted (as in later discussions) for simplicity. Equation (6) is temporally discretized using a finite difference method in order to perform numerical calculations. We confirmed that solutions to the resultant simultaneous equations always exist, because the tridiagonal matrix is diagonally dominant (see Appendix B). The simultaneous equations are solved easily using LU decomposition. In the next section, these simultaneous equations with time evolution are solved using numerical methods.

\section{Results of numerical simulations}

\subsection{Numerical calculations}

It is assumed that the initial state of the mucus under each segment is $\sigma_{n}=1$ (elasticity), because the animals are adhering to the substratum when the locomotion begins. We can also estimate the scale of some physical quantities from previous research ${ }^{6,7,20,36}$ (see Supplement). First, the order of $\mu$ is estimated to be about $10^{-3}$. In addition, that of $F_{u}$ is also estimated to be about $10^{-3}$ for $W=1.0$. In our simulations, we divided the foot into 50 segments $(N=50)$, which is sufficient to propagate a wave. The dimensionless parameters of the physical features are set as follows: $W=1.0$, $\alpha=0.5$, and $\mu=0.005$. The parameter $F_{u}$ ranges from $10^{-3}$ to $10^{-2}$.

Figure B.4(a) shows chronological samples and Figure B.4(c) shows the spatiotemporal dynamics of a simulation using $F_{u}=0.35 \times 10^{-2}, F_{l}=0.001 \times$ $10^{-2}, \kappa=1.0$, and $q=0.005$. The mucus under each segment changes its state (viscosity or elasticity) from moment to moment due to the contraction and expansion of muscle. From the results in Figures B.4(a) and B.4(c), it seems that segments of expanded muscle are anchored. In other words, the 
regions of contracted muscle move along the body.

Figure B.4(b) shows chronological samples, and Figure B.4(d) shows the spatiotemporal dynamics of a simulation using $F_{u}=0.42 \times 10^{-2}, F_{l}=0.001 \times$ $10^{-2}, \kappa=1.0$, and $q=0.005$. That is, the only change from Figures B.4(a) and B.4(c) is the value of $F_{u}$. Figure B.4(d) shows with perfect clarity that retrograde wave locomotion is achieved. In this case, it seems that anchoring occurs around the regions of muscle contraction in Figure B.4(b). Hence, the regions of muscle expansion move along the body. Figure B.4(e) is the result of plotting each phase of the natural length of the RTS when each segment is anchored. This result shows that a small change in the yield point can greatly change the phase of anchoring.

Figure B.5 shows the temporal alteration of a segment located in the middle of the body. The upper graphs show the average natural lengths of both sides of the RTS of the 25th segment, $l_{25-1 / 2}$ and $l_{25+1 / 2}$, and its average actual length. The lower graphs show the total force acting on the 25th segment, $F_{25+1 / 2}-F_{25-1 / 2}$. Panels (a) and (b) represent the cases of direct waves and retrograde waves, respectively. The anchoring clearly occurred in the elongated state of the spring in (a). By contrast, the anchoring occurred in the contracted state of the spring in (b). Although the force generated by one side increases when the difference between natural length and actual length increases, a segment is not able to move without a difference on both sides. Note that the mucus always yields from the left side of the body in the case of either a direct wave or a retrograde wave, as can be seen in Figures B.4(a) and B.4(b). Hence, it is reasonable to focus on the spring between the 24th and 25th segments when the mucus under the 24th segment 
yields and becomes fluid. The spring is in the contracted state in the case of a direct wave and the elongated state in the case of a retrograde wave. It is found that the propulsive force is generated by pushing in the case of a direct wave. Conversely, the propulsive force is generated by pulling in the case of a retrograde wave. In a direct wave, the jump (B) of the hysteresis loop shown in Figure B.2(c) occurs at the positive value $F_{u}$, as confirmed by the graph of force in Figure B.5(a). Moreover, because the total force is positive when the segment is not anchored, the whole body can move rightward. By contrast, in a retrograde wave, the jump (B) of the hysteresis loop occurs at the negative value $-F_{u}$, as confirmed by Figure B.5(b). As the total force is negative when the segment is not anchored, the whole body can move leftward.

Figure B.6 is a velocity diagram that shows in detail the effect that some properties of mucus have on motion. Here, the velocity ratio $R$ is calculated as the ratio of the velocity of the center of the body to the wave velocity. Based on previous knowledge ${ }^{20,36}$, we define an efficient directional motion as when the absolute value of $R$ is more than 0.1. We see in Figure B.6(a) that there exist parametric regions of $F_{u}$ and $F_{l}$ for achieving valid locomotion. Figure B.6(a) clearly shows that regions for both direct and retrograde waves exist. That is, Figure B.6(a) indicates that both $F_{u}$ and $F_{l}$ are properties of mucus that influence the motion of the body. In addition, this study examines the influence of the viscosity of mucus on locomotion. Figure B.6(b) is the result of calculations of $R$ with respect to the viscosity of mucus $\mu$ for $F_{u}=0.45 \times 10^{-2}, F_{l}=0.0005 \times 10^{-2}, \kappa=1.0$, and $q=0.005$. We found that $\mu$ makes a significant contribution to locomotion. Our results indicate that 
the direct wave appears when $\mu$ is either below a lower threshold or above an upper threshold. Comparing these two regions of the direct wave, $R$ is larger when $\mu$ is smaller. The region of the retrograde wave exists between the two regions of the direct wave. The diagram shows that the direction of the body depends strongly on the viscosity of the mucus. The relation between the velocity ratio $R$ and the average of the anchoring phase can be seen in Figure B.6(c). The natural length is greater (elongation) when the phase $(P=t-2 \pi W n / N)$ satisfies $\sin P>0$. Conversely, the natural length is less (contraction) when $\sin P<0$. The results show that the anchoring phase occurs in the state of elongation when the direct wave is chosen, and in the state of contraction when the retrograde wave is chosen (i.e., almost in antiphase with respect to the direct wave).

Now, we investigate the influence of muscle features on the style of locomotion. Biologically, it would be an important finding if the physical features of muscle were to have implications for determining the direction of the body. Figure B.7(a) therefore shows the effects of muscle parameters. The parameter $\kappa$ corresponds to the stiffness of an active spring, and $\alpha$ represents the ratio of contraction and elongation. Figure B.7(a) illustrates that there is a parametric region of $\kappa$ and $\alpha$ in which locomotion can be achieved by crawling. Furthermore, the nature of the muscle clearly affects the direction of movement. Figure B.7(b), which is similar to Figure B.6(c), shows the result of changing the anchoring phase while varying the parameters $\kappa$ and $\alpha$. The two styles of locomotion are realized by changing the anchoring phase in response to changes in the parameters. Moreover, the black lines in Figure B.7(a) plot the range of the spring coefficient $r_{s c}\left(=2 \kappa \alpha /\left(1-\alpha^{2}\right)\right)$; i.e., 
the difference between the maximum and minimum values of the spring coefficient. The black lines are only drawn when valid locomotion is achieved; that is, when $0.8 \leq r_{s c} \leq 5.2$. We observe that when $r_{s c}$ is relatively small, $0.8 \leq r_{s c} \leq 2.8$, effective locomotion can be realized by means of the retrograde wave as well as the direct wave. When $r_{s c}$ is relatively large, $4.0 \leq r_{s c}$, the direct wave is the only feasible strategy.

\section{Discussion and conclusions}

The aim of this paper has been to theoretically verify that mutual interaction between muscular contraction and mucus can realize adhesive locomotion. Therefore, we built a mathematical model with two essential assumptions. The first was the active spring (RTS) and the multi-segment body for mimicking the propagation of a muscular wave, and the second was the hysteresis loop for describing changes in the nature of mucus. Our numerical simulations demonstrated that propulsive force adequate for locomotion can be generated by the interaction of muscular wave propagation and viscoelastic mucus. Under this mechanism, the mucus has a role in controlling the friction with the ground.

We first verified that the crawling locomotion is validly simulated by the mechanism: that is, by the mutual interaction between periodic muscular waves of the soft foot and the specific properties of the mucus. At first, the muscle begins to contract and expand periodically, in unison with the phase of the internal oscillators. The mucus under each segment is pulled and pushed depending on the tension induced by the muscular waves. When the tension (force) acting on the mucus becomes larger, the mucus changes to 
a viscous liquid from an elastic solid working to fix the body to the substrate (anchoring). Beyond this point, the body segments can move smoothly (slipping). Both anchoring and slipping regions exist along the foot. Like the muscular wave, these anchoring and slipping regions propagate.

Furthermore, two styles of locomotion, the direct wave and the retrograde wave, are obtained in our numerical simulations. The simple mechanical difference between direct and retrograde waves was clarified by Jones and Trueman ${ }^{4}$ after Lissmann ${ }^{13}$ and Miller ${ }^{1,15}$. The simplified mechanism of direct waves is as follows: the foot adheres to the substratum at maximum extension; then, forward movement occurs when the foot is longitudinally contracted and the contracted portion propagates forward. That is, migration of the center of gravity occurs in the contracted portion of the foot. In contrast, the mechanism of retrograde waves is as follows: the foot is attached along its length at its minimum extension; then, the foot is maximally extended forward and the extended portion propagates backward. That is, migration of the center of gravity occurs in the extended portion of the foot. These previous mechanisms were not fundamental mechanisms but merely described the appearance of each style of locomotion based on observations. Our numerical results represent an important progression, as these existing mechanisms were reproduced by considering the underlying principles.

Comparing the results of a mathematical model for peristaltic locomotion $^{34}$ makes it easy to understand why these two styles of locomotion can occur. Tanaka et al. ${ }^{34}$ claimed that the peristaltic locomotion of both the direct wave and the retrograde wave can be realized if the phase of anchoring can be changed. Thus, in the simplest terms, it is necessary to vary the 
anchoring phase in order to realize two styles of locomotion. Based on our numerical calculations, we claim that it is possible to change the anchor phase by changing only the properties of the mucus (Figures B.4(e) and B.6(c)) or the features of the muscle (Figure B.7(b)). Our study fully supports the conclusions of Tanaka et al. However, we can also say that active control of friction is not required in the case of spontaneous friction transitions by mucus, unlike in the mechanism of peristaltic locomotion.

Figure 4 explains why the anchoring phase would change. What is significant here is the condition of the foot when the jump (B) in Figure B.2(c) occurs; i.e., the moment at which the mucus changes from elastic to viscous. The force can be generated by pushing as well as pulling. In the case of a direct wave, the jump (B) occurs in the segment of foot being pushed. Hence, the mucus becomes a viscous liquid before this portion of foot reaches its full contraction. Therefore, the elongated portions of the foot are anchored. By contrast, in the case of a retrograde wave, the jump (B) occurs in the segment of foot being pulled, and the mucus changes to a viscous liquid before this portion of foot reaches its full elongation. That is, the contracted portions of the foot are anchored.

Basically, the choice between two styles is uniquely determined by the parameters $F_{l}$ and $F_{u}$. However, we already know from our additional numerical calculations that these two styles of locomotion coexist in the boundary regions between direct and retrograde waves, as shown in Figures B.6(a), B.6(b), and B.7(a). In this study, $\sigma_{n}$ was initially set to 1 (elasticity) for all $n$, because this is the condition in nature. Under this assumption, there exist parametric regions where the style of locomotion suddenly changes. It 
would be reasonable to consider that there are no such boundaries for parametric regions of actual animals. From a mathematical perspective, however, the question is why the sign of the velocity reverses immediately when some parameter values are changed. This problem, which originates from the existence of multiple solutions, should be analyzed by improving the description of the mucus from a discrete model to a continuous model, and by decreasing the degrees of freedom of the mass system to three. We will investigate this phenomenon, as a part of future work, by conducting a nonlinear stability analysis.

Finally, let us now consider our results in the context of the research by Chan et al. ${ }^{21}$. Our study partially supports their results, in that the locomotion by direct wave can be achieved through interaction between muscular contractions and mucus. However, they concluded that spontaneous friction transitions by mucus cannot produce locomotion by retrograde wave. Although we cannot say exactly why their conclusion differs from ours, we suggest a possibility based on Figure B.7(a). That is, their model (and robots) may impose the condition that the value of either $\alpha$ or $r_{s c}$ is large. If this is true, we are in agreement with the result that only the direct wave is obtained. In their study, a mollusk-like flexible body might not have been considered, because they focused on the properties of mucus. They overlooked a significant factor: the properties of muscle contribute significantly to the choice of movement direction in the mechanism of spontaneous friction transitions by mucus. The two styles of locomotion may be realizable if a robot is made on the basis of our results. Our study would then serve as a guideline for the development of such robots. 
In this study, one deficiency in our mathematical model is the assumption that the recovery is the jump (D) shown in Figure B.2(c). Actually, the recovery of elasticity should be performed slowly in comparison with jump B. It is not yet clear how long the mucus needs to recover its elasticity. As future work, we will build a mathematical model that considers a healing time in the recovery process. Through an improved model, we will be able to investigate how the recovery time affects the locomotion, and how long a time can be valid for effective locomotion.

This study reinforces the assertion that mucus has an important role in friction control for adhesive locomotion. Furthermore, we have obtained new insights into the nature of how the mucus and muscles directly affect the style of locomotion. Future experimental research into the yield point of mucus and the ratio of muscle contraction in various species, as well as mathematical investigations into energy efficiency, will reveal the background of the evolutionary acquisition of locomotion strategies in gastropods and related species.

\section{Acknowledgements}

We would like to thank Atsushi Tero and Masakazu Akiyama for meaningful discussions about the mechanism of crawling locomotion by mucus in the early stages of this study. This study was supported by the Meiji University Global COE Program "Formation and Development of Mathematical Sciences Based on Modeling and Analysis" and by the Core Research for Evolutional Science and Technology (CREST) program "Alliance for Breakthroughs between Mathematics and Sciences" of the Japan Science and Tech- 
nology Agency (JST).

\section{Appendix A. Device for mimicking a flexible muscle} an idea has already been proposed in a mathematical model of movement for slime mold ${ }^{37}$ and earthworms ${ }^{34}$. Moreover, analogous devices have been developed in robotics, such as the real-time tunable spring (RTS) pioneered by Umedachi et al..$^{35}$.

There are two types of active springs that change in natural length: one is a hinged spring, as assumed in the model of Tanaka et al. ${ }^{34}$, and the other is a reeling spring with a motor, as used in this study. Essentially, there is not much difference between these two devices, because each is self-driven and describes a flexible body. The difference lies in the way that the natural length is changed. In the case of a hinged spring, only the apparent length is changed (by opening the angle of the hinge). By contrast, in the case of a reeling spring with a motor, the actual length is changed. The description of the force generated by a spring differs between hinged springs and reeled springs, even if the natural lengths $l(t)[\mathrm{m}]$ are similarly controlled as follows:

$$
l(t)=l_{0}(1+\alpha \sin \omega t),
$$


where $l_{0}[\mathrm{~m}], \alpha, \omega[\mathrm{Hz}]$, and $t[\mathrm{~s}]$ represent the average natural length, contraction ratio, wave frequency, and time, respectively. In the case of a hinged spring, the lengths of parts of the spring do not change, even though the apparent length does change. Hence, the force $F_{h}[\mathrm{~N}]$ acting on segments generated by the spring is described as follows:

$$
F_{h}(t)=\kappa_{h}\left(x_{1}-x_{0}-l(t)\right)
$$

where $x_{0}[\mathrm{~m}]$ and $x_{1}[\mathrm{~m}]$ are the position of segments connected by the hinged spring. In this case, $\kappa_{h}[\mathrm{~N} / \mathrm{m}]$ represents the spring constant.

In the case of a reeling spring with a motor, the force is slightly different from that in the case of a hinged spring. In general, a spring becomes softer as the natural length becomes greater. Therefore, in the case where the natural length can vary, it is reasonable to suppose that the spring constant will vary. That is, the spring constant will be inversely proportional to the natural length. Hence, the force $F_{r}[\mathrm{~N}]$ generated by the spring is described as follows:

$$
F_{r}(t)=\frac{\kappa_{r}}{l(t)}\left(x_{1}-x_{0}-l(t)\right) .
$$

In this case, $\kappa_{r}[\mathrm{~N}]$ represents the stiffness of the spring and is determined by its materials.

\section{Appendix B. Formulas for numerical calculations}

To perform the numerical calculations, the dimensionless equation (6) is discretized using a finite difference method that makes no assumption as to 


$$
\begin{aligned}
(1- & \left.\sigma_{i}^{j}\right) \mu \frac{x_{i}^{j}-x_{i}^{j-1}}{\delta t}+\sigma_{i}^{j}\left(x_{i}^{j}-\bar{x}_{i}^{j}\right) \\
= & \frac{\kappa_{i+\frac{1}{2}}}{l_{i+\frac{1}{2}}}\left(x_{i+1}^{j}-x_{i}^{j}-l_{i+\frac{1}{2}}\right)+q_{i+\frac{1}{2}}\left(\frac{x_{i+1}^{j}-x_{i+1}^{j-1}}{\delta t}-\frac{x_{i}^{j}-x_{i}^{j-1}}{\delta t}\right) \\
& \quad-\frac{\kappa_{i-\frac{1}{2}}}{l_{i-\frac{1}{2}}}\left(x_{i}^{j}-x_{i-1}^{j}-l_{i-\frac{1}{2}}\right)-q_{i-\frac{1}{2}}\left(\frac{x_{i}^{j}-x_{i}^{j-1}}{\delta t}-\frac{x_{i-1}^{j}-x_{i-1}^{j-1}}{\delta t}\right),
\end{aligned}
$$

${ }_{465}$ where $\delta t$ denotes a time step and $x_{i}^{j}$ is the position of the $i$ th segment equation (B.1) in the case of $i \neq 0, N$ :

$$
\begin{aligned}
-\left(\frac{\kappa_{i+\frac{1}{2}}}{l_{i+\frac{1}{2}}}+\frac{q_{i+\frac{1}{2}}}{\delta t}\right) x_{i+1}^{j} & \\
+\left(\sigma_{i}^{j}+\right. & \left.\frac{\left(1-\sigma_{i}^{j}\right) \mu+q_{i+\frac{1}{2}}+q_{i-\frac{1}{2}}}{\delta t}+\frac{\kappa_{i+\frac{1}{2}}}{l_{i+\frac{1}{2}}}+\frac{\kappa_{i-\frac{1}{2}}}{l_{i-\frac{1}{2}}}\right) x_{i}^{j} \\
& -\left(\frac{\kappa_{i-\frac{1}{2}}}{l_{i-\frac{1}{2}}}+\frac{q_{i-\frac{1}{2}}}{\delta t}\right) x_{i-1}^{j} \\
=-\kappa_{i+\frac{1}{2}}+\kappa_{i-\frac{1}{2}} & +\sigma_{i}^{j} \bar{x}_{i}^{j}-\frac{q_{i+\frac{1}{2}}}{\delta t} x_{i+1}^{j-1} \\
& +\frac{\left(1-\sigma_{i}^{j}\right) \mu+q_{i+\frac{1}{2}}+q_{i-\frac{1}{2}}}{\delta t} x_{i}^{j-1}-\frac{q_{i-\frac{1}{2}}}{\delta t} x_{i-1}^{j-1} .
\end{aligned}
$$
end-points of the body are given as follows: 


$$
\begin{gathered}
-\left(\frac{\kappa_{0+\frac{1}{2}}}{l_{0+\frac{1}{2}}}+\frac{q_{0+\frac{1}{2}}}{\delta t}\right) x_{1}^{j}+\left(\sigma_{0}^{j}+\frac{\left(1-\sigma_{0}^{j}\right) \mu+q_{0+\frac{1}{2}}}{\delta t}+\frac{\kappa_{0+\frac{1}{2}}}{l_{0+\frac{1}{2}}}\right) x_{0}^{j} \\
=-\kappa_{0+\frac{1}{2}}+\sigma_{0}^{j} \bar{x}_{0}^{j}-\frac{q_{0+\frac{1}{2}}}{\delta t} x_{1}^{j-1}+\frac{\left(1-\sigma_{0}^{j}\right) \mu+q_{0+\frac{1}{2}}}{\delta t} x_{0}^{j-1} . \\
\left(\sigma_{N}^{j}+\frac{\left(1-\sigma_{N}^{j}\right) \mu+q_{N-\frac{1}{2}}}{\delta t}+\frac{\kappa_{N-\frac{1}{2}}}{l_{N-\frac{1}{2}}}\right) x_{N}^{j}-\left(\frac{\kappa_{N-\frac{1}{2}}}{l_{N-\frac{1}{2}}}+\frac{q_{N-\frac{1}{2}}}{\delta t}\right) x_{N-1}^{j} \\
=\kappa_{N-\frac{1}{2}}+\sigma_{N}^{j} \bar{x}_{N}^{j}+\frac{\left(1-\sigma_{N}^{j}\right) \mu+q_{N-\frac{1}{2}}}{\delta t} x_{N}^{j-1}-\frac{q_{N-\frac{1}{2}}}{\delta t} x_{N-1}^{j-1} .
\end{gathered}
$$

Solutions of these simultaneous equations always exist, because the tridiagonal matrix is diagonally dominant.

\section{References}

[1] S.L. Miller, The classification, taxonomic distribution, and evolution of locomotor types among prosobranch gastropods, Proc. Malac. Soc. Lond. 41 (1974) 233-261.

[2] H.W. Lissmann, The mechanism of locomotion in gastropod molluscs. I. Kinematic, J. Exp. Biol. 21 (1945) 58-69.

[3] J. Gray, Animal Locomotion, George Weidenfeld and Nicolson Ltd., London, 1968.

[4] H.D. Jones, E.R. Trueman, Locomotion of the limpet, Patella vulgata L., J. Exp. Biol. 52 (1970) 201-216.

[5] Y. Grimm-Jorgensen, M.E. Ducor, J. Piscatelli, Surface mucus production in gastropods is dependent on environmental salinity and humidity, Comp. Biochem. Physiol. 83A(3) (1986) 415-419. 
[6] M.W. Denny, The role of gastropod pedal mucus in locomotion, Nature 285 (1980) 160-161. (doi:10.1038/285160a0)

[7] M.W. Denny, J.M. Gosline, The physical properties of the pedal mucus of the terrestrial slug, Ariolimax columbianus, J. Exp. Biol. 88 (1980) 375-393.

[8] M.W. Denny, Locomotion: the cost of gastropod crawling, Science $\mathbf{2 0 8}$ (1980) 1288-1290.

[9] M.W. Denny, A quantitative model for the adhesive locomotion of the terrestrial slug, Ariolimax columbianus, J. Exp. Biol. 91 (1981) 195-217.

[10] G.H. Parker, The pedal locomotion of the sea-hare Aplysia californica, J. Exp. Zool. 24 (1917) 139-145.

[11] R.A. Barr, Some observations on the pedal gland of Milax, Q. J. Microsc. Sci. 70 (1926) 647-667.

[12] R.A. Barr, Some notes on the mucous and skin glands of Arion ater, $Q$. J. Microsc. Sci. 71 (1927) 503-525.

[13] H.W. Lissmann, The mechanism of locomotion in gastropod molluscs. II. Kinetics, J. Exp. Biol. 22 (1945) 37-50.

[14] H.D. Jones, The mechanism of locomotion in Agriolimax reticulatus (Mollusca; Gastropoda), J. Zool., Land. 171 (1973) 489-498.

[15] S.L. Miller, Adaptive design of locomotion and foot form in prosobranch gastropods, J. exp. Biol. Ecol. 14 (1974) 99-156. 
[16] E.R. Trueman, Locomotion in gastropods, Mollusca 4 (1983) 155-198.

[17] R.M. Alexander, Exploring Biomechanics: Animals in Motion, Freeman and Company, New York, NY, 1992.

[18] D.A. Donovan, T.H. Carefoot, Locomotion in the abalone Haliotis kamtschatkana: pedal morphology and cost of transport, J. Exp. Biol. 200 (1997) 1145-1153.

[19] R.M. Alexander, Principles of Animal Locomotion, Princeton University Press, Princeton, NJ, 2002, pp. 86-90, 166-180.

[20] J.H. Lai, J.C. del Alamo, J. Rodríguez-Rodríguez, J.C. Lasheras, The mechanics of the adhesive locomotion of terrestrial gastropods, J. Exp. Biol. 213 (2010) 3920-3933.

[21] B. Chan, N.J. Balmforth, A.E. Hosoi, Building a better snail: lubrication and adhesive locomotion, Phys. Fluids 17 (2005) 113101.

[22] E. Lauga, A.E. Hosoi, Tuning gastropod locomotion: modeling the influence of mucus rheology on the cost of crawling, Phys. Fluids 18 (2006) 113102. (doi:10.1063/ 1.2382591)

[23] R.H. Ewoldt, C. Clasen, A. E. Hosoi, G.H. McKinley, Rheological fingerprinting of gastropod pedal mucus and synthetic complex fluids for biomimicking adhesive locomotion, Soft Matter 3 (2007) 634-643.

[24] H. Simroth, Die bewegung unserer landschnecken, hauptsächlich erörtert an der sohle des, Limax. Z. Wiss. Zool. 22 (1879) 284. 
[25] M. Lister, Exercitatio Anatomica: in qua de Cochleis, maximè terrestribus et Limacibus, agitur, Sumptibus Sam. Smith \& Benj. Walford, London, 1694.

[26] G.H. Parker, The mechanism of locomotion in gastropods, J. Morphol. 22 (1911) 155-170.

[27] R. Dubois, F. Vles, Locomotion des gasteropodes, Compt. Rend. Acad. Sci. Paris 144 (1907) 658-659.

[28] J. Gray, H.W. Lissmann, Studies in locomotion. VII. Locomotory reflexes in the earthworm, J. Exp. Biol. 15 (1938) 506-517.

[29] K. Zimmermann, I. Zeidis, C. Behn, Mechanics of Terrestrial Locomotion - With a Focus on Non-pedal Motion Systems, Springer, Berlin, 2009.

[30] N. Bolotnik, M. Pivovarov, I. Zeidis, K. Zimmermann, The undulatory motion of a chain of particles in a resistive medium, ZAMM 91(4) (2011) 259-275.

[31] F.L. Chernous'ko, Analysis and optimization of the rectilinear motion of two-body system, Appl. Math. Mech. 75(5) (2011), 493-500.

[32] J. Steigenberger, C. Behn, Worm-Like Locomotion Systems - An Intermediate Theoretical Approach, Oldenbourg, Munich, 2012.

[33] K. Zimmermann, I. Zeidis, M. Pivovarov, Dynamics of two interconnected mass points in a resistant medium, Differ. Equ. Dyn. Syst. 21 (2013) 21-28. 
[34] Y. Tanaka, K. Ito, T. Nakagaki, R. Kobayashi, Mechanics of peristaltic locomotion and role of anchoring, J. R. Soc. Interface 9 (2011) 222-233.

[35] T. Umedachi, Y. Yamada, A. Ishiguro, Development of a real-time tunable spring - toward independent control of position and stiffness of joints, J. Robo. Mech. 19 (2007) 27-33.

[36] Based on our observations, $R$ of Japanese abalone (Haliotis discus discus Reeve (ditaxic direct wave)) satisfies $R \approx 1 / 7(\approx 0.143)$.

[37] R. Kobayashi, A. Tero, T. Nakagaki, Mathematical model for rhythmic protoplasmic movement in the true slime mold, J. Math. Biol. 53 (2006) $273-286$.

(a)

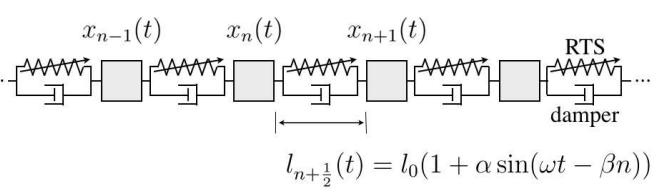

(b)

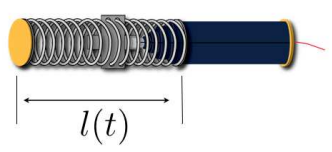

Figure B.1: Modeling of the ventral foot of gastropods. (a) Multi-segment model that generates muscular wave propagation. Each segment is connected by a real-time tunable spring (RTS) and a damper. (b) Image sketch of RTS $^{35}$ for simple mimicking of a muscle. 
(a)

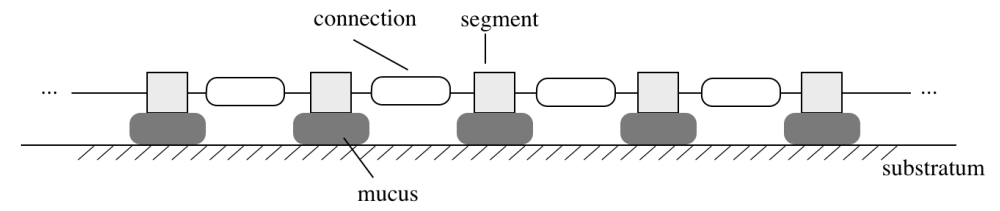

(b)

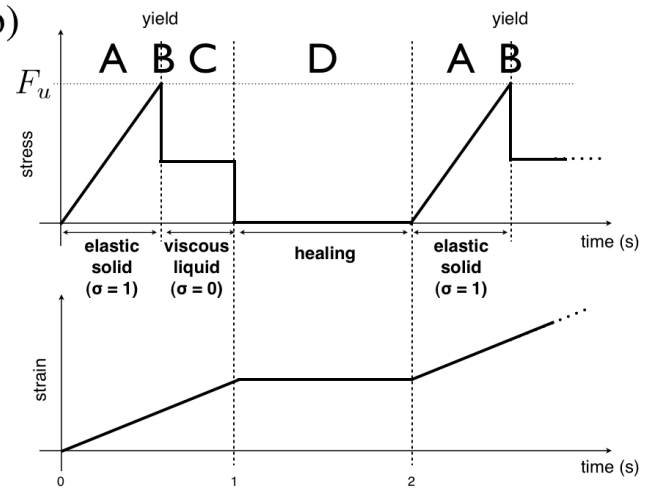

(c)

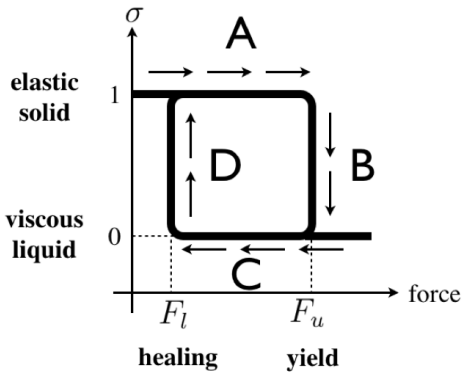

Figure B.2: Modeling of pedal mucus. (a) Pedal mucus under each segment has two states: elastic solid and viscous liquid. (b) Outline of one of the results in the experiments of Denny and Gosline ${ }^{6,7}$. The parameter $F_{u}$ corresponds to yield stress, and this is the threshold at which the property of the mucus changes from elasticity to viscosity. The stages A, B, C, and D correspond to those in a hysteresis loop, respectively. (c) Internal hysteresis loop of pedal mucus. The parameter $F_{l}$ corresponds to the stress when the mucus is healing, and this is the threshold of the change from viscosity to elasticity. The variable $\sigma$ represents the state of the mucus. When $\sigma=1$, the mucus behaves as an elastic solid. If the external force on a segment increases (A) and reaches $F_{u}$, the mucus yields (B) and behaves as a viscous liquid with $\sigma=0$. If the external force on a segment then decreases (C) below $F_{l}$, the mucus recovers its elasticity (D) and has $\sigma=1$. 


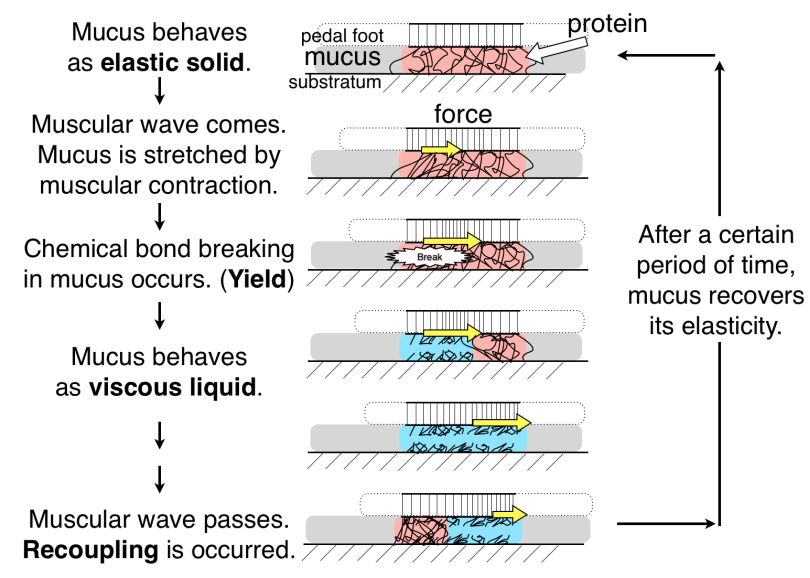

Figure B.3: Image sketch of mucus dynamics under stress. 


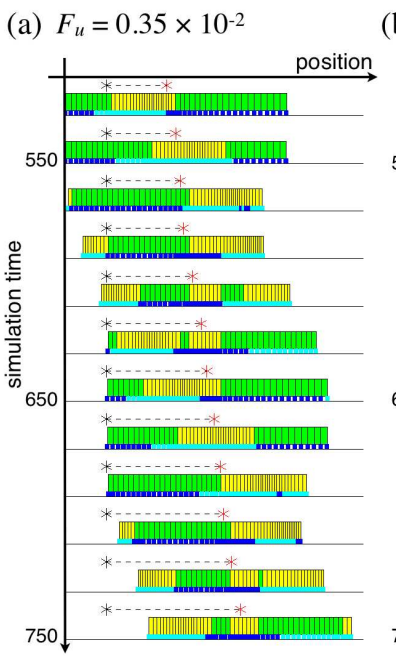

(b) $F_{u}=0.42 \times 10^{-2}$

(c) $F_{u}=0.35 \times 10^{-2}$
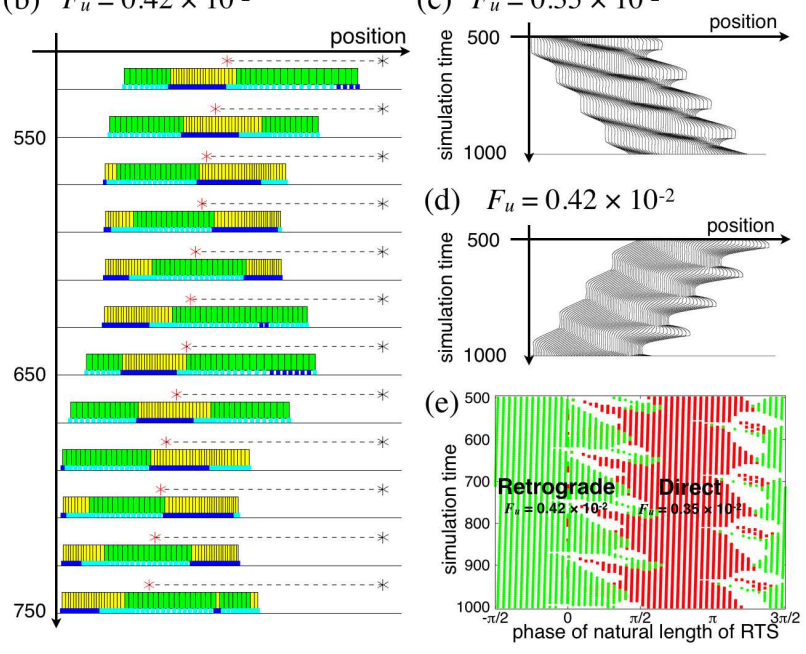

Figure B.4: Results of simulations with our model. (a), (b) Chronological samples of simulations. In each foot segment, the green regions are expanded muscle and the yellow regions are contracted muscle in comparison to the average length of the RTS. In the mucus under each foot segment, the light blue regions are viscous liquid (slipping) and the dark blue regions are elastic solid (anchoring). The black and red asterisks show the positions of the center at the initial time and subsequent times, respectively. (a) Direct wave for $F_{u}=0.35 \times 10^{-2}, F_{l}=0.001 \times 10^{-2}, \kappa=1.0$, and $q=0.005$. The waves of contraction propagate in the positive direction and the whole body moves in the same direction. (b) Retrograde wave for $F_{u}=0.42 \times 10^{-2}, F_{l}=0.001 \times 10^{-2}, \kappa=1.0$, and $q=0.005$. The waves of expansion propagate in the positive direction, but the whole body moves in the opposite direction. (c), (d) Time series plots of the position of each segment using the same parameters as for (a) and (b). (e) Phase plots of $l(t)$, the natural length of the RTS, when the mucus behaves as an elastic solid (anchoring); i.e., the phase of the adhesive region of the foot is indicated. The red stripes are the results with the same parameters as for (a), and the green stripes are with the same parameters as for (b). 

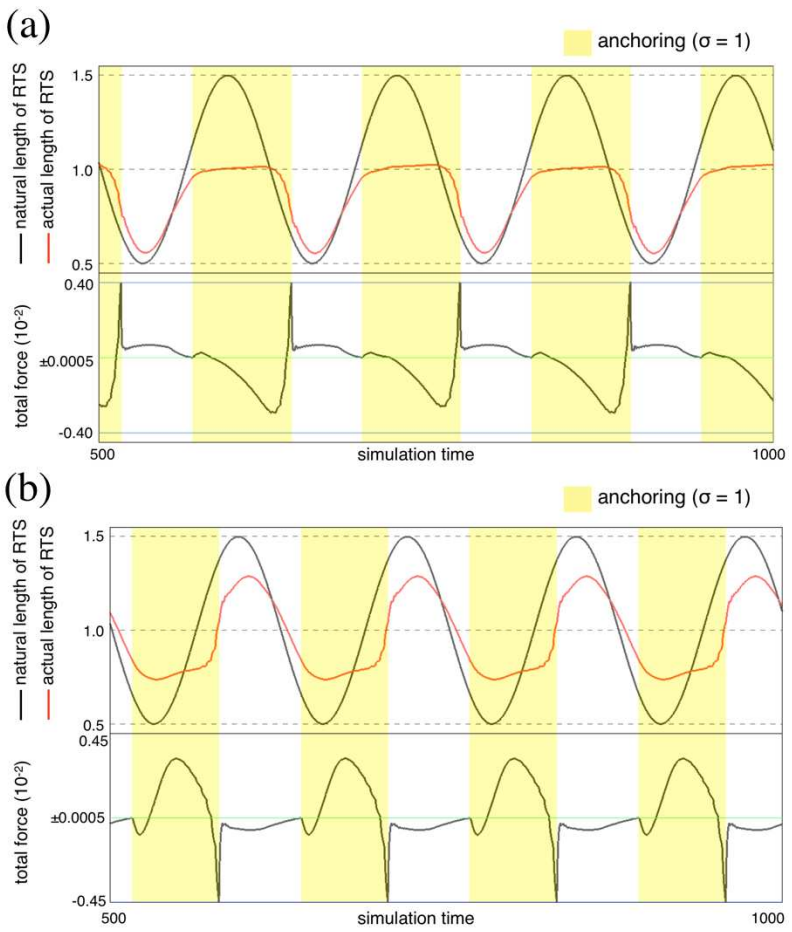

Figure B.5: Temporal plots of natural length and actual length of RTS (upper) and force acting on the 25th segment (lower). The yellow regions correspond to the times of anchoring; i.e., the times when the state of the mucus under the 25 th segment was an elastic solid $(\sigma=1)$. (a) Direct wave $\left(F_{u}=0.40 \times 10^{-2}, F_{l}=0.0005 \times 10^{-2}, \kappa=2.0\right.$, and $q=0.05$ ). The jump (B) of mucus in the hysteresis loop, as shown in Figure B.2(c), occurs when the force becomes greater than $F_{u}$. (b) Retrograde wave $\left(F_{u}=0.45 \times 10^{-2}\right.$, $F_{l}=0.0005 \times 10^{-2}, \kappa=2.0$, and $q=0.05$ ). The jump (B) occurs when the force becomes less than $-F_{u}$. 

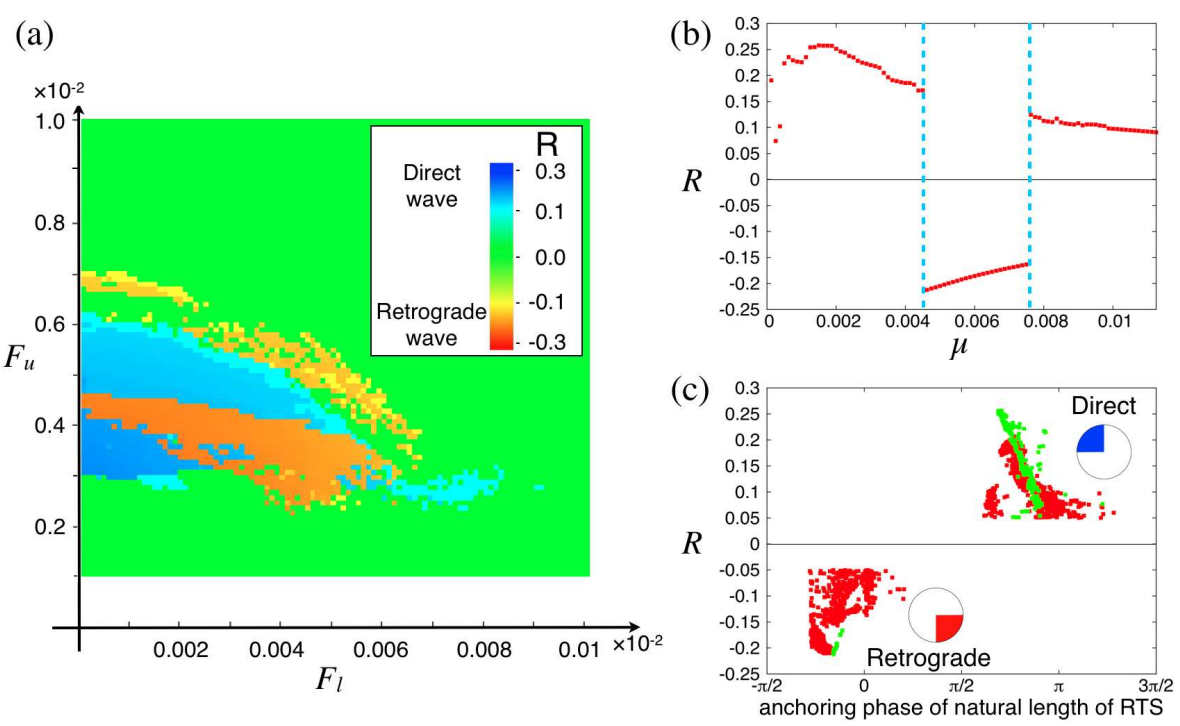

Figure B.6: Effects of properties of mucus on the velocity ratio $R$ for $\kappa=1.0$ and $q=0.005$.

(a) Color plot of $R$ with respect to $F_{u}$ and $F_{l}$. If the absolute value of $R$ is greater than 0.1 , the region is colored blue for a direct wave $(R \geq 0.1)$ or red for a retrograde wave $(R \leq-0.1)$. (b) Plots of $R$ with respect to the viscosity of mucus $\mu$. (c) Relation between the anchoring phase and $R$ in the case where the absolute value of $R$ is greater than 0.05 . The red plots are obtained by varying $F_{u}$ and $F_{l}$, whereas the green plots are obtained by varying $\mu$. 

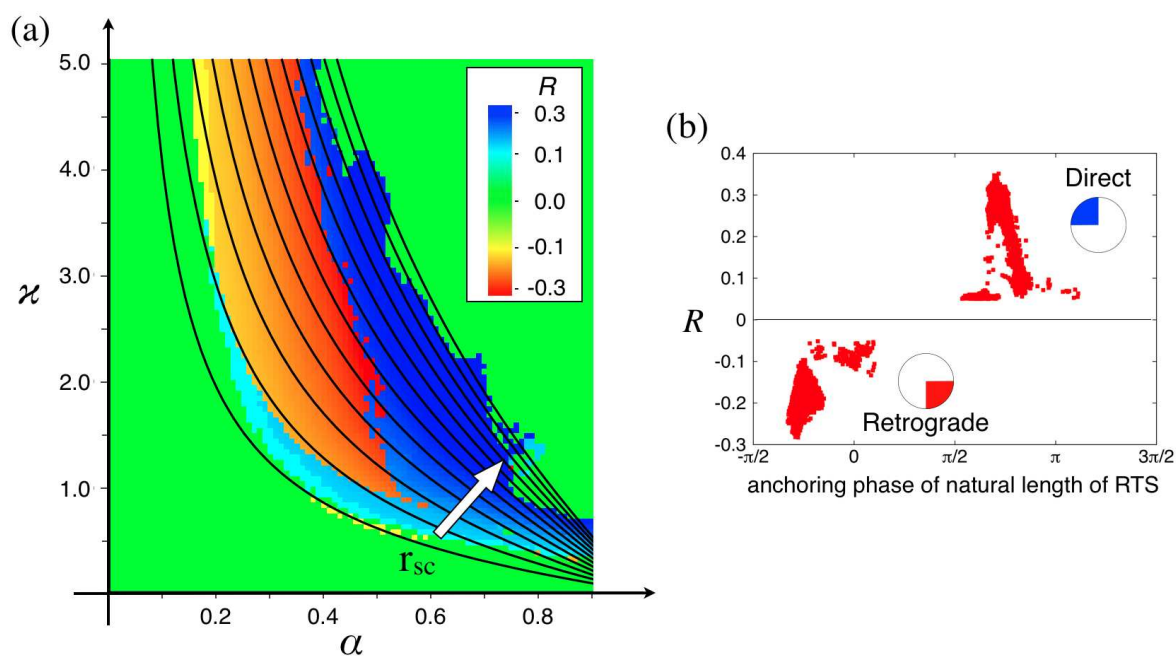

Figure B.7: Effect of muscle features on velocity ratio $R$. (a) Color plots of $R$ with respect to $\kappa$ and $\alpha$. The result is for $F_{u}=0.45 \times 10^{-2}, F_{l}=0.0005 \times 10^{-2}$, and $q=0.005$. If the absolute value of $R$ is greater than 0.1 , the region is colored blue for a direct wave $(R \geq 0.1)$ or red for a retrograde wave $(R \leq-0.1)$. The black lines are plots of $r_{s c}$, the range of the spring coefficient of the RTS, and the white arrow indicates the positive direction. The range plotted with the black lines is $0.8 \leq r_{s c} \leq 5.2$. (b) Relation between the average anchoring phase and $R$ in the case where the absolute value of $R$ is greater than 0.05 . The plots show results obtained by varying $\kappa$ and $\alpha$. 


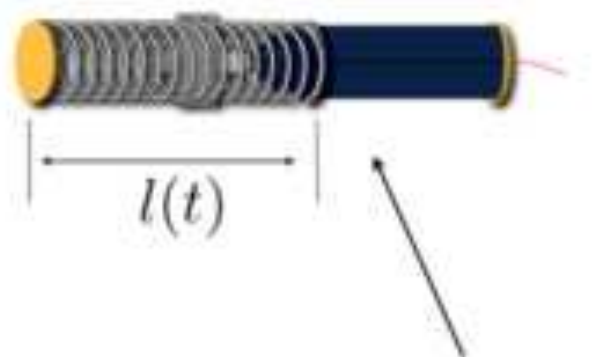

pedal foot

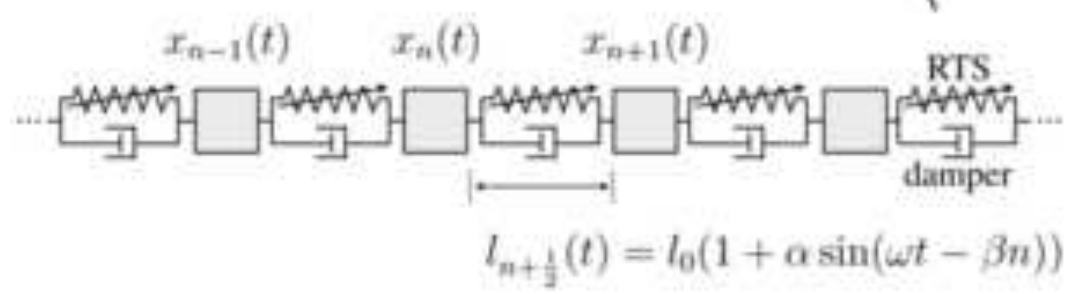

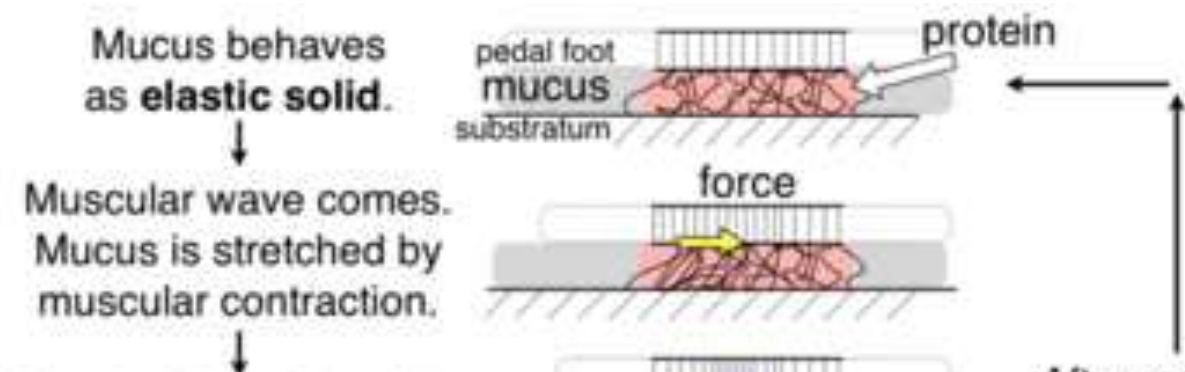

Chemical bond breaking in mucus occurs. (Yield)

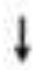

Mucus behaves as viscous liquid.

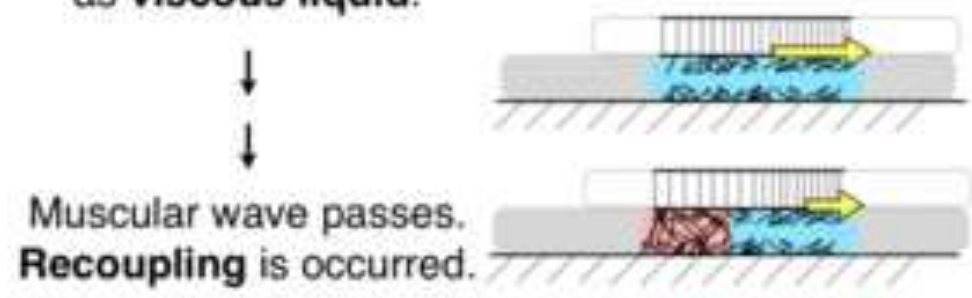

Muscular wave passes.

Recoupling is occurred.
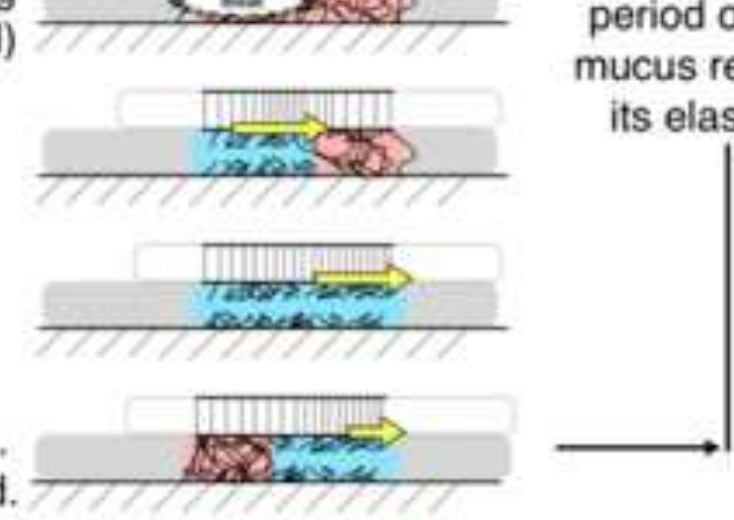

After a certain period of time, mucus recovers its elasticity.

$$
\mid
$$

